\title{
LE PROBLÈME DES BÉNÉFICES DISTRIBUABLES EXAMINÉ À LA LUMIERE DU THÉOREME DE M. DE FINETTI
}

\author{
J. ADAM \\ Bruxelles
}

\begin{abstract}
Résuméé
Le théorème de de Finetti relatif à la ruine des joueurs peut constıtuer un modèle mathématique susceptible de décrire l'activité de l'assureur. Cette propriété est mise à profit pour poser, sur un plan général, le problème de l'acceptation des risques et celui de bénéfices distrıbuables. Est envisagé ensuite le cas particulier d'un portefeuille composé de contrats identiques dont le risque est une variable de Poisson composée au sens de Feller. Les variations du bénéfice distribuable avec le plein (si l'assureur pratique la réassurance excess-loss) et avec la fortune propre y sont étudiées et le cas où la distribution conditionnelle des sinistres est log. normale est également examiné.
\end{abstract}

\section{Préambule}

Le problème de l'utilisation des soldes techniques établis à la fin de chaque exercice comptable est certainement une des importantes questions qu'ait à résoudre un assureur.

En effet, il y a lieu, à ce moment, de définir ce que l'on affectera aux réserves de sécurité et ce que l'on distribuera immédiatement, que ce soit en rémunération du capital pour les actionnaires ou en „participation aux bénéfices” pour les assurés.

La solution, purement actuarielle, que nous évoquons dans cette note pour résoudre ce problème, repose sur le théorème de $M$. de Finetti et s'inspire étroitement des idées développées par M. Dubourdieu dans son ouvrage consacré à la ,,Théorie du risque dans les assurances de répartition" ${ }^{1}$ ).

\section{Le théorème de $M$. de Finetti}

Si un joueur (en l'occurrence un assureur) engage, avec une fortune initiale $Z$, une suite indéfinie de paris tels que son gain algébrique $y$ dans l'un quelconque de ces paris vérifie la condition

$$
E\left\{e^{U(x)-U(x+Y)}\right\}=\mathrm{x}
$$

1) Gauthier-Villars, Paris, I952. 
où $x$ désigne le montant de sa fortune au moment où il engage le pari en question et $U(x)$ une fonction croissante de $x$, la probabilité pour qu'il finisse un jour ou l'autre par se ruiner est au plus égale à

$$
\Theta=e^{U(0)-U(Z)} .
$$

Nous utiliserons ce théorème dans le cas où l'on choisi pour $U(x)$ une expression linéaire: $U(x)=R x$ avec $R$ positif. Il en résulte alors que si l'assureur accepte des contrats à la condition que, pour chacun, le gain algébrique $Y^{1}$ ) satisfasse à la relation

$$
E\left\{e^{-R Y}\right\}=\mathrm{I} \text {, }
$$

les chances qu'a le ,fonds de risque ${ }^{2}$ ) d'être épuisé un jour, ou l'autre, sont inférieures ou égale à

$$
\theta=e^{-R Z},
$$

$Z$ étant le niveau initial du fonds.

Comme nous le verrons dans les paragraphes suivants, ce théorème remarquable possède une grande valeur heuristique, car il peut constituer une véritable assise technique de 'activité d'assureur, base sur laquelle repose la solution des problèmes importants d'acceptation de risques, de bénéfices distribuables et de réserves de sécurité.

\section{Le problème de l'acceptation des risques}

Cette question capitale peut être solutionnée élégamment par le théorème de $\mathrm{M}$. de Finetti, pourvu, bien entendu, que l'on connaisse le risque, c'est-à-dire la fonction de distribution du montant total $X$ des sinistres susceptibles de survenir pendant la période de couverture au contrat soumis à l'acceptation.

1) Le gain algébrique associé à un contrat est la différence entre la ,,prime de risque" et les sinistres.

Nous entendons par ,prime de risque" celle qui est destinée à couvrir les sinistres, c'est-à-dire la prime pure majorée du chargement de sécurité.

2) Le ,fonds de risque" est un compte fictif qui est crédité des primes de risque relatives aux contrats en portefeuille et débité des sinistres correspondants. 
Les relations (2.3) et (2.4) constitueront à cet égard un véritable critère d'acceptation, en permettant de calculer la prime de risque qui correspond à une fortune propre donnée $Z$ et à une limite supérieure $\theta$ donnée de la probabilité de ruine.

En effet, si $P$ et $\beta$ représentent respectivement la prime pure et le taux de chargement de sécurité de notre contrat, le gain algébrique s'écrira

$$
Y=(\mathrm{x}+\beta) P-X,
$$

et la condition (2.3)

$$
E\left\{e^{-R Y}\right\}=E\left\{e^{-R[(1+\beta) P-X]}\right\}
$$

donnera

$$
(\mathrm{I}+\beta) P=\frac{\log \varphi(R)}{R} \quad \text { où } \varphi(R)=E\left\{e^{R X}\right\} .
$$

Le paramètre $R$, appelé habituellement ,indice de sécurité” du contrat, est fourni par (2.4):

$$
R=\frac{k}{Z} \quad \text { avec } k=\log \frac{\mathrm{I}}{\theta} .
$$

En fait, sur le plan économique, ce n'est pas l'aspect tarification qui est le plus important dans le problème de l'acceptation; ce qui compte c'est de pouvoir modifier, soit par des franchises, soit par la réassurance, la distribution du risque à supporter, pour le faire correspondre, par le jeu de (2.6), à la prime de risque compatible avec les conditions du marché.

\section{Le problème des bénéfices distribuables}

L'assureur, disposant au départ d'une fortune $Z$ et ayant fixé la limite $\theta$ de la probabilité de ruine à un niveau négligeable, est pratiquement assuré de faire face à ses obligations techniques, s'il alimente le ,,fonds de risque" par des primes satisfaisant à la relation (2.6) dans laquelle $\varphi(R)$ est la fonction caractéristique ${ }^{1}$ ) calculée en $R=k / Z$ (voir 2.7) de la variable aléatoire $X$, qui représente le risque réellement supporté par le fonds.

1) Dans la pratique, la variable $X$ est bornée, si bien que $\varphi(R)$ est toujours défini. 
La question qui se pose maintenant est de savoir si, pour un portefeuille donné, le solde entre les primes brutes encaissées et les primes de risque dont il est question ci-dessus est suffisant pour permettre à l'assureur de couvrir les frais généraux et distribuer des bénéfices.

Dans la négative, quel procédé faut-il employer pour rendre ce solde suffisant et même pour l'optimiser?

Le problème étant posé sur le plan général, nous essaierons de le solutionner ne fût-ce que partiellement, dans le cadre du cas particulier défini au paragraphe suivant.

\section{Cas particulier}

Considérons un portefeuille composé d'un ensemble de contrats annuels identiques ${ }^{1}$ ) pour chacun desquels le montant total $X$ des sinistres susceptibles de se produire pendant l'année envisagée est représenté par une variable de Poisson composée au sens de Feller (compound Poisson Variable).

Alors $X$ peut se mettre sous la forme

$$
X=\sum_{i=0}^{N} H_{i},\left(H_{0}=0\right),
$$

où $N$, représentant le nombre des sinistres surceptibles de survenir pendant l'année, est distribué suivant une loi de Poisson simple de paramètre $\chi: P(k)=P(N=k)=e^{-x} \frac{\chi^{k}}{k !} ; \chi>0 ; k=0, \mathrm{I}, 2, \ldots$ et où les $H_{1}, H_{2}, \ldots$, représentant les montants des différents sinistres, sont des variables aléatoires indépendantes et identiquement distribuées avec une fonction de répartition (conditionnelle) $V(x) . X$ a comme fonction de distribution

$$
\begin{gathered}
G(x)=P[X \leqslant x]=\sum_{k=0}^{\infty} e^{-x} \frac{\chi^{k}}{k !} V^{* k}(x), \quad x \geqslant 0, V^{* 0}(x)=\mathbf{I} \\
=0, x \leqslant 0
\end{gathered}
$$

et comme fonction caractéristique

$$
\varphi(R)=\int_{0}^{\infty} e^{R x} d G(x)=e^{x[\psi(R)-1]}
$$

1) Pour fixer les idées nous supposerons qu'il s'agit de contrats d'assurance de dommages à garantie illimitée. 
avec

$$
\Psi(R)=\int_{0}^{\infty} e^{R x} d V(x) .
$$

En portant cette expression de $\varphi(R)$ dans (2.6), le critère d'acceptation devient

$$
(\mathrm{I}+\beta) P=\frac{\chi}{R}[\Psi(R)-\mathrm{I}]
$$

On notera en passant que (5.I) n'est rien d'autre que l'expression célèbre de la théorie collective de risque,

$$
\mathrm{I}+(\mathrm{I}+\beta) R=\int_{0}^{\infty} e^{R x} d V(r)
$$

dans laquelle on a pris pour unité le coût moyen d'un sinistre

$$
\left(\int_{0}^{\infty} x d V(x)=\mathrm{I}\right) \text {. }
$$

En effet, si, dans (5.I), nous remplaçons $P$ par $\chi^{m}$ avec $m=$ $=\int_{0}^{\infty} x d V(r)$, il vient $\mathrm{I}+(\mathrm{I}+\beta) R m=\Psi(R)=\int_{0}^{\infty} e^{R x} d V(r),(\mathrm{ex}-$ pression dans laquelle, bien sûr, $m$ n'est pas pris pour unité).

Si la relation (5.I), appliquée avec une fonction de répartition $V(x)$ qui est celle des sinistres auxquels l'assuré est exposé, fournit une prime de risque impratiquable sur le marché, l'assureur devra modifier le risque supporté par son fonds. Cette modification, qui se fait par la réassurance quand c'est possible, a pour but de procurer à l'assureur une marge bénéficiaire positive et même optimum sur la prime brute du marché.

Cómme nous le verrons plus loin, cette marge constitue un bénéfice libre distribuable immédiatement, bien distinct d'un solde de compte d'exploitation courant.

Nous ne rechercherons pas ici la meilleure forme de réassurance qui peut solutionner notre problème ${ }^{1}$ ); nous nous placerons simplement dans les conditions les plus courantes, qui sont celles de la

1) On trouvera dans le bulletin de l'Association Royale des actuaires belges $\mathrm{n}^{0}$ 60 (I960) un article de M. H. Lambert intitulé ,Un eapplication de la théorie collective du risque: la réassurance" qui traite de l'efficacité des différentes formes de réassurance. 
réassurance excess-loss, pour étudier les variations du bénéfice distribuable avec le plein et la fortune propre de l'assureur.

Explicitons maintenant ce bénéfice $B$ d'un contrat quelconque au moyen des données analytiques du problème.

Partons de la prime brute

avec

$$
P^{\prime \prime}=(\mathrm{I}+K) P=(\mathrm{I}+K) \chi^{m}
$$

$$
m=\int_{0}^{\infty} x d V(x)
$$

et considérons les divers éléments qu'il faut retrancher de cette prime pour obtenir le solde à distribuer.

Il y a tout d'abord la prime de risque $\left.\left(\mathrm{I}+\beta_{c}\right) P_{c}{ }^{1}\right)$ que la cédante doit verser dans son fonds de risque. Cette prime est définie par (5.I) qui s'écrit, en exprimant $R$ au moyen de (2.7)

$$
\left(\mathrm{I}+\beta_{c}\right) P_{c}=\frac{\chi Z}{k}\left[\Psi_{c}\left(\frac{k}{Z}, n\right)-\mathrm{I}\right]
$$

avec, si $n$ désigne le plein,

$$
\begin{gathered}
P_{c}=\chi m_{c}(n) \\
m_{c}(n)=\int_{0}^{n} x d V(r)+n \int_{n}^{\infty} d V(x)
\end{gathered}
$$

et

$$
\Psi_{c}\left(\frac{k}{Z}, n\right)=\int_{0}^{n} e^{(k / Z) x} d V(x)+e^{(k / Z) n} \int_{n}^{\infty} d V(x) .
$$

Ensuite, la prime destinée au réassureur; nous l'écrirons

$$
\left[\mathrm{I}+\lambda_{r}(n)\right] P_{r}=\left[\mathrm{I}+\lambda_{r}(n)\right] \chi^{m_{r}}(n)
$$

avec

$$
m_{r}(n)=\int_{n}^{\infty}(x-n) d V(x)
$$

$\lambda_{r}(n)$ est le taux de chargement, net de commission de réassurance, demandé par le réassureur. En général ce taux est fonction du plein, cependant, dans nos développements, nous le supposerons constant ${ }^{2}$ ).

1) Nous utilisons l'indice $c$ pour les éléments relatifs à la cédante et l'indice $r$ pour les éléments relatifs du réassureur.

$\left.{ }^{2}\right)$ Cette hypothèse est fort restrictive, nous en convenons, et ne correspond pas à la réalité. Une manière de la lever est d'imaginer que le réassureur agit ,rationnellement" au sens de la relation (5.I), c'est-à-dire qu'il calcule sa prime de risque selon la formule 
Pour finir, il y a les commissions, que nous mettrons sous la forme $\gamma P$ et les frais généraux, que nous écrirons $\varphi P$. Le taux $\varphi$ comprenant la part des frais de la cédante due à la réassurance, est en général une fonction de $n$ que nous supposerons constante dans la suite.

Si l'on pose $\eta=K-\gamma-\varphi$, le bénéfice distribuable s'écrira ${ }^{1}$ )

$$
B=(\mathrm{I}+\eta) P-\left(\mathrm{x}+\lambda_{r}\right) P_{r}-\left(\mathrm{I}+\beta_{c}\right) P_{c},
$$

c'est-à-dire, en tenant compte de (5.3) et (5.7)

$$
B=\chi\left\{(\mathrm{I}+\eta) m-\left(\mathrm{I}+\lambda_{r}\right) m_{r}(n)-\frac{Z}{k}\left[\Psi_{c}\left(\frac{k}{Z}, n\right)-\mathrm{I}\right]\right\}
$$

\section{Bénéfice distribuable et réserve de sécurité}

Nous avons déjà signalé le caractère fictif du ,fonds de risque" car en pratique la comptabilité d'un tel fonds ne se tient pas. Ce que l'on détermine à la fin de chaque exercice c'est le gain algébrique d'exploitation $G$ qui, pour chaque contrat de notre portefeuille, est donné par

$$
G=(\mathrm{I}+\eta) P-\left(\mathrm{I}+\lambda_{r}\right) P_{r}-X_{c},
$$

où $X_{c}$ représente le montant total des sinistres (réserves comprises), net de reassurance, à charge de la cédante.

Comme le résultat $S$ du ,fonds de risque" vaut, pour chaque contrat,

$$
S=\left(\mathrm{I}+\beta_{c}\right) P_{c}-X_{c},
$$

on a, d'après $(5.8)$ et (6.I),

$$
G=B+S \text {. }
$$

avec

$$
\left(\mathrm{I}+\lambda_{r}^{*}\right) P_{r}=\frac{\chi Z_{r}}{k_{r}}\left[\Psi_{r}\left(\frac{k_{r}}{Z_{r}}, n\right)-\mathrm{I}\right],
$$

$$
\Psi_{r}\left(\frac{k_{r}}{Z_{r}}, n\right)=\int_{0}^{n} d V(x)+\int_{n}^{\infty} e^{\frac{k_{r} r}{Z_{r}}(x-n)} d V(x)
$$

et qu'il y ajoute, pour frais et bénéfice, un chargement $\Delta \lambda . P_{r}$ dont le taux $\Delta \lambda$ est une fonction connue de $n$ ou une constante. Encore faut-il que la cédante connaisse $Z_{r}, k_{r}$ et $\Delta \lambda$.

1) Nous négligeons ici les intérêts. 
Cette dernière relation permet en fait d'utiliser judicieusement le solde technique $\Sigma G$ procuré à la fin de l'exercice comptable par tous les contrats en portefeuille. En effet, l'application répétée des relations (5.8) ou (5.9) fournit le montant $\Sigma B$ du bénéfice libre que l'assureur peut distribuer, tandis que le reliquat, positif ou négatif, $\Sigma G-\Sigma B$, doit alimenter, en plus ou en moins, la réserve de sécurité.

$\mathrm{Si}$, par exemple, le portefeuille considéré donne un solde technique de 10.000.000 et que les bénéfices libres s'élèvent à 8.000.000, la réserve de sécurité augmentera alors de 2.000.000. Par contre, si le solde comptable ne s'élève qu'à 5.000 .000 , c'est 3.000 .000 qu'il faudra puiser dans la réserve de sécurité pour assurer le service du bénéfice.

\section{Variation du bénéfice distribuable avec le plein}

Nous allons considérer $B$ comme une fonction de $n$ uniquement, dans les hypothèses restrictives où $\lambda_{r}$ et $\eta$ sont indépendants de $n$.

Lorsque $n=0$, c'est-à-dire, lorsqu'il y a cession intégrale du risque au réassureur, l'expression (5.9), dans laquelle $\Psi_{c}$ et $m_{r}$ sont donnés respectivement en (5.6) et (5.7), devient

$$
B(0)=\chi\left(\eta-\lambda_{r}\right) m \text {. }
$$

Dans ces conditions, la cédante ne pourra distribuer un bénéfice que si le taux de chargement résiduel $\eta$ excède le taux de chargement net de commission $\lambda_{r}$ demandé par le réassureur.

Si $n=\infty$, c'est-à-dire s'il n'y a pas de réassurance, on a

$$
B(\infty)=\chi\left\{(\mathrm{I}+\eta) m-\frac{Z}{k}\left[\int_{0}^{\infty} e^{(k / Z) x} d V(x)-\mathrm{I}\right]\right\}
$$

En se référant à (5.I), on peut encore écrire

$$
B(\infty)=\chi(\eta-\beta) m \text {, }
$$

où $\beta$ est le taux de chargement de risque nécessité par le critère d'acceptation (5.I) dans le cas où l'assureur couvre les risques sans avoir recours à la réassurance.

Calculons maintenant la dérivée de $B$ par rapport à $n$ :

$$
\frac{d B}{d n}=-\chi\left[\left(\mathrm{I}+\lambda_{r}\right) \frac{d m_{r}}{d n}+\frac{Z}{k} \frac{d \Psi_{c}}{d n}\right]
$$


Comme

$$
\frac{d m_{r}}{d n}=-\int_{n}^{\infty} d V(x)
$$

et que

$$
\frac{d \Psi_{c}}{d n}=\frac{k}{Z} e^{(k / Z) n} \int_{n}^{\infty} d V(x)
$$

il vient

$$
\frac{d B}{d n}=\chi\left[I+\lambda_{r}-e^{(k / Z) n}\right] \int_{n}^{\infty} d V(x)
$$

Cette expression s'annule pour

$$
\left.n=n_{0}=\frac{Z}{k} \log \left(\mathrm{I}+\lambda_{r}\right),{ }^{1}\right)
$$

est positive lorsque $n<n_{0}$ et négative lorsque $n>n_{0}$.

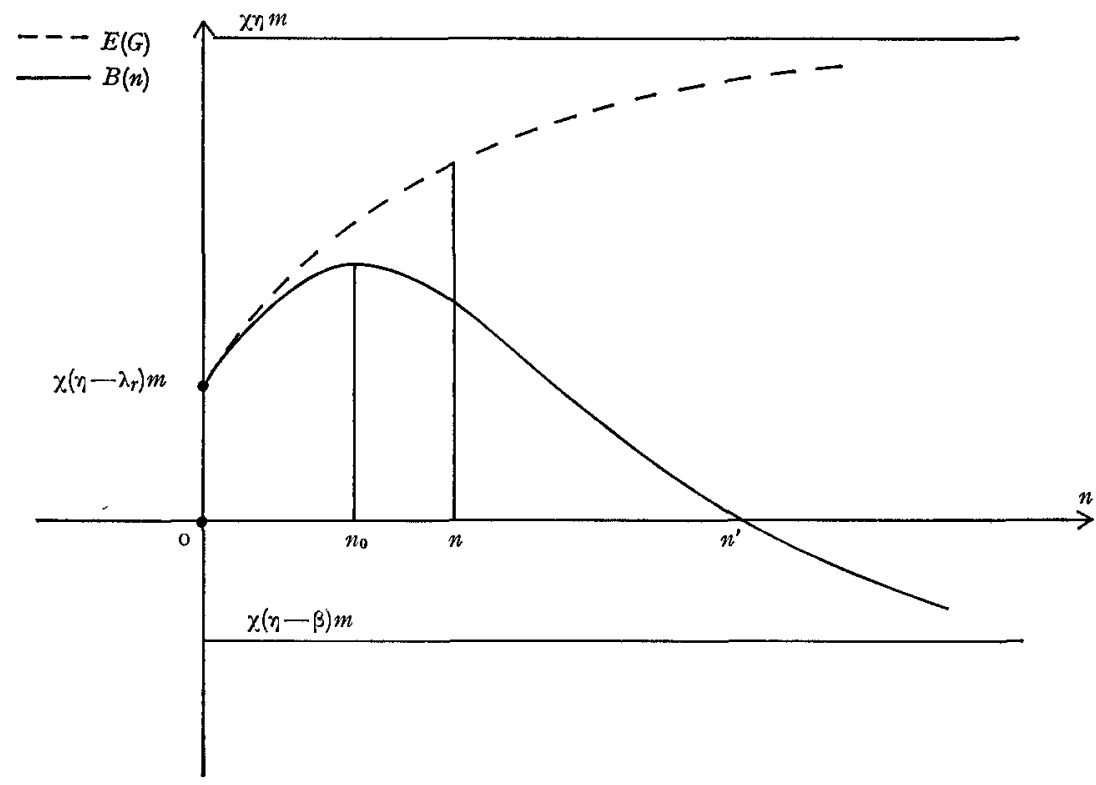

1) Relation que l'on retrouve dans le cadre de la théorie collective du risque (voir Monsieur H. Lambert, op. cit.) 
La courbe en trait plein du graphique ci-contre illustre une allure possible de la fonction $B(n)$.

Cette courbe montre qu'il y aurait intérêt à ne pas dépasser un plein maximum $n^{\prime}$ et qu'il existe un plein optimum $n_{0}$ auquel, d'ailleurs, l'assureur n'est pas tenu de se conformer, s'il estime, comme le montre la courbe en trait brisé donnant $E(G)$ en fonction de $n$, qu'il a intérêt à choisir un plein un peu supérieur dans le but d'augmenter $E(G)$ même au détriment de $B$.

En se reportant à (6.3) on a en effet

$$
E(G)=B+E(S) \text {. }
$$

Comme

$$
\begin{gathered}
E(S)=\beta_{c} P_{c}, \quad \text { il vient } \\
E(G)=B+\beta_{c} P_{c},
\end{gathered}
$$

ou encore, d'après $(5.8)$

Pour

$$
\begin{gathered}
E(G)=\eta P-\lambda_{r} P_{r}=\chi\left(\eta m-\lambda_{r} m_{r}(n)\right) . \\
n=0, E(G)=B=\chi\left(\eta-\lambda_{r}\right) m
\end{gathered}
$$

et pour

$$
\text { D'autre part } \frac{d E(G)}{d n}=\chi \lambda_{r} \int_{n}^{\infty} d V(x),
$$

expression qui est positive et qui décroit avec $n$

$$
\text { Pour } n=0, \frac{d E(G)}{d n}=\frac{d B}{d n}=\chi \lambda_{r} .
$$

\section{Variation du bénéfice distribuable avec la fortune propre}

La dérivation de (5.9) par rapport à $Z$ donne

$$
\frac{d B}{d Z}=\frac{\chi}{k}\left[\mathbf{I}-\Psi_{c}-Z \frac{d \Psi_{c}}{d Z}\right] .
$$

Pour ne pas alourdir l'écriture, mettons l'expression (5.6) de $\Psi_{c}$ sous la forme

$$
\Psi_{c}=E\left\{e^{(k / Z) x_{c}}\right\}
$$

où $X_{c}$ représente le coût d'un sinistre, pour la cédante.

En développant l'exponentielle en série, nous aurons, si $E\left\{X_{c}\right\}$ existe pour tout entier non négatif $v$,

$$
\Psi_{c}^{v}=E\left\{\sum_{\nu=0}^{\infty} \frac{I}{\nu !}\left(\frac{k}{Z}\right)^{\nu} X_{c}^{\nu}\right\}=\sum_{\nu=0}^{\infty} \frac{I}{\nu !}\left(\frac{k}{Z}\right) E\left\{X_{c}^{\nu}\right\}
$$


et

$$
\frac{d \Psi_{c}}{d Z}=-\frac{\mathrm{I}}{Z} \sum_{\nu=1}^{\infty} \frac{\mathrm{I}}{(\nu-\mathrm{I}) !}\left(\frac{k}{Z}\right)^{\nu} E\left(X_{c}^{\nu}\right) .
$$

Maintenant (8.I) s'écrit

$$
\frac{d B}{d Z}=\frac{\chi}{k} \sum_{\nu=1}^{\infty}\left(\frac{k}{Z}\right)^{\nu} E\left(X_{c}^{\nu}\right)\left(\frac{\mathrm{I}}{(\nu-\mathrm{I}) !}-\frac{\mathrm{I}}{\nu !}\right)
$$

Cette expression étant positive, le bénéfice distribuable est une fonction croissante de la fortune initiale.

Pour $Z=0$, on observera que $B=-\infty$. En effet, en se reportant à (5.9), on voit que le terme en $Z$ du second membre

$-\frac{Z}{k}\left[\Psi_{c}-\mathrm{I}\right]=-\frac{Z}{k}\left[E\left(e^{\left.(k / Z) X_{\bullet}\right)}-\mathrm{I}\right]=-\sum_{v=1}^{\infty} \frac{\mathrm{I}}{v !}\left(\frac{k}{Z}\right)^{v-1} E\left(X_{c}\right)^{\nu}\right.$,

tend vers $-\infty$ quand $Z$ tend vers 0 .

Pour $Z$ tendant vers $\infty$, cette dernière expression tend vers $-E\left(X_{c}\right)=-m_{c}(n)$

et la limite de $B$ a pour expression

$$
\chi\left(\eta m-\lambda_{r} m_{r}(n)=E(G) .\right.
$$

Remarquons que le bénéfice optimum $B_{0}$, c'est-à-dire l'expression (5.9) dans laquelle $n$ est donné par (7.6), est une fonction croissante de $Z$.

En effet

$$
\begin{aligned}
\frac{d B_{0}}{d Z} & =\frac{\partial B_{0}}{\partial Z}+\frac{\partial B_{0}}{\partial n_{0}} \frac{d n_{0}}{d Z} \\
& =\frac{\partial B_{0}}{\partial Z}>0,
\end{aligned}
$$

puisque

$$
\frac{\partial B_{0}}{\partial n_{0}}=0 .
$$

Ce résultat très intéressant montre, entre autres, que si 2 assureurs travaillent dans les conditions optimum définies par (7.6), avec la même limite supérieure $\theta$ de la probabilité de ruine, mais avec des 
fortunes initiales respectives $Z_{1}$ et $Z_{2}, Z_{2}$ étant supérieur à $Z_{1}$, le bénéfice distribuable optimum de $A_{2}$ sera supérieur à celui de $A_{1}$.

On peut même ajouter que ces deux assureurs auraient intérêt à s'associer, c'est-à-dire à mettre en commun leurs fortunes respectives $Z_{1}$ et $Z_{2}$. Nous allons illustrer cela par un exemple.

\section{Exemple}

Nous allons calculer quelques valeurs du bénéfice distribuable optimum $B_{0}$ dans l'hypothèse où $V(x)$ est log-normale.

$$
\frac{d V(x)}{d x}=v(x)=\frac{\mathrm{I}}{\sigma x \sqrt{2 \pi}} e^{-1 / 2 A^{2}(x)}, x \geqslant 0
$$

avec

$$
A(x)=\frac{\log x-m}{\sigma} .
$$

Considérons d'abord trois intégrales-types qui interviennent dans le calcul de $B_{0}$ à savoir:

$$
\begin{gathered}
\mathrm{I}(n)=\int_{n}^{\infty} v(x) d x, \\
\mathrm{II}(n)=\int_{n}^{\infty} x v(x) d x, \\
\text { III }(n)=\int_{0}^{n} e^{(k / Z) x} v(x) d x . \\
\mathrm{I}(n)=\int_{n}^{\infty} v(x) d x=\frac{\mathrm{I}}{\sigma \sqrt{2 \pi}} \int_{u}^{\infty} \frac{\mathrm{I}}{x} e^{-1 / 2 A^{2}(x)} d x \\
=\frac{\mathrm{I}}{\sqrt{2 \pi}} \int_{A(n)}^{\infty} e^{-1 / 2 x^{2}} d x=\mathrm{I}-\phi[A(n)] . \\
\mathrm{II}(n)=\int_{n}^{\infty} x v(x) d x=\frac{\mathrm{I}}{\sigma \sqrt{2 \pi}} \int_{n}^{\infty} e^{-1 / 2 A^{2}(x)} d x \\
=\frac{e^{m}}{\sqrt{2 \pi}} \int_{A(n)}^{\infty} e^{\sigma x-1 / 2 x^{2}} d x .
\end{gathered}
$$


En remarquant que $\sigma x-\frac{1}{2} x^{2}=\frac{1}{2} \sigma^{2}-\frac{1}{2}(x-\sigma)^{2}$, on a

$$
\begin{gathered}
\text { II }(n)=\frac{e^{m+1 / 2 \sigma^{2}}}{\sqrt{2 \pi}} \int_{A(n)}^{\infty} e^{-1 / 2(x-\sigma)^{2}} d x \\
=\frac{e^{m+1 / 2 \sigma^{2}}}{\sqrt{2 \pi}} \int_{A(n)-\sigma}^{\infty} e^{-1 / 2 x^{2}} d x \\
=e^{m+1 / 2 \sigma^{2}}\{\mathrm{I}-\phi[A(n)-\sigma]\} \\
\operatorname{III}(n)=\int_{0}^{n} e^{k / Z} v(x) d x=\frac{\mathrm{I}}{\sigma \sqrt{2 \pi}} \int_{0}^{n} \frac{e^{(k / Z) x}}{x} e^{-1 / 2 A^{2}(x)} d x \\
=\frac{\mathrm{I}}{\sigma \sqrt{2 \pi}} \sum_{\nu=0}^{\infty} \frac{(k / Z)^{v}}{v !} \int_{0}^{n} x^{\nu-1} e^{-1 / 2 A^{2}(x)} d x \\
=\frac{\mathrm{I}}{\sqrt{2 \pi}} \sum_{\nu=0}^{\infty} \frac{(k / Z)^{v} e^{m \nu}}{\nu !} \int_{-\infty}^{A(n)} e^{v \sigma x-1 / 2 x^{2}} d x
\end{gathered}
$$

Remarquons que $v \sigma x-\frac{1}{2} x^{2}=\frac{1}{2} v^{2} \sigma^{2}-\frac{1}{2}(x-v \sigma)^{2}$.

Il vient alors

$$
\text { III } \begin{aligned}
(n) & =\frac{I}{\sqrt{2 \pi}} \sum_{\nu=0}^{\infty} \frac{(k / Z)^{v} e^{m v+1 / 2 v^{2} \sigma^{2}}}{\nu !} \int_{-\infty}^{A(n)} e^{-1 / 2(x-v \sigma)^{2}} d x \\
& =\sum_{\nu=0}^{\infty} \frac{(k / Z)^{v}}{\nu !} e^{m v+1 / 2 v^{v^{2} \sigma^{2}} \phi[A(n)-v \sigma]^{1)}}
\end{aligned}
$$

Ces intégrales étant déterminées, on obtiendra la valeur de $B_{0}$ par la formule

$$
\begin{array}{r}
B^{c}=\chi\left\{(\mathrm{I}+\eta) e^{m+1 / 2 \sigma^{2}}-\left(\mathrm{I}+\lambda_{r}\right)\left[\mathrm{II}\left(n_{0}\right)-n_{0} \mathrm{I}\left(n_{0}\right)\right]-\right. \\
\left.-\frac{Z}{k}\left[\mathrm{III}\left(\mathrm{n}_{0}\right)+\left(\mathrm{I}+\lambda_{r}\right) \mathrm{I}\left(n_{0}\right)-\mathrm{I}\right]\right\}
\end{array}
$$

1) $e^{m v+1} / 2^{v^{2}} \sigma^{2}$ est le moment ordinaire de l'ordre $v$ de la distribution lognormale. 
Supposons maintenant que 2 assureurs travaillent dans les conditions suivantes, identiques pour chacun d'eux:

$$
\begin{gathered}
m=7,5 ; \sigma=2 ; \chi=0,25 \text { par an, } \theta=\mathrm{I0}^{-3}, \\
\eta=0,05, Z=10.000 .000 \text { et } \lambda_{r}=0, \text { Io. }
\end{gathered}
$$

Le plein optimum (7.6) sera pour chacun d'eux de 137.976 et le bénéfice distribuable correspondant (9.4) s'élèvera annuellement à I8,45 par contrat ${ }^{1}$ ).

Si ces 2 assureurs s'associent en mettant en commun leurs capitaux $(Z=20.000 .000)$, nous aurons, toutes autres conditions restant inchangées, un plein optimum de 275.95I (double du précédent car $\lambda_{r}$ n'a pas changé par hypothèse) et un bénéfice annuel distribuable de 9323 par contrat, qui représente environ 5 fois le précédent. Ce bénéfice tombe à $5 \mathrm{I}, 84$, soit $\approx 2,8 \times \mathrm{I} 8,45$, si le chargement $\lambda_{r}$ est porté à 0,20 .

Dans ce cas, le plein optimum doit être fixé à 527.875 .

Ces calculs ne sont donnés ici qu'à titre indicatif, dans le but de montrer l'intérêt de la question des bénéfices distribuables.

Août I97o.

1) Etant donné le peu de précision des tables utilisées pour calculer $\phi$ nous nous sommes limités au zème terme de la série III $(n)$, qui converge d'ailleurs très rapidement. Un surcroît de précision pour $\phi$ serait cependant nécessaire vu l'importance du facteur $Z / k$ dans la pratique. 